\title{
Diagnóstico de programa de evaluación, metodologías, indicadores y evaluación de resultados: caso de organismos autónomos del estado de Guanajuato
}

\section{Evaluation program, methodologies, indicators and evaluation of results Analysis: Guanajuato public companys}

\author{
GALVÁN-ZAVALA Karina†, JIMÉNEZ-RICO, Artemio y REA-CHAVEZ, Luis Gerardo
}

Universidad de Guanajuato, División de Ciencias Económico-Administrativas, Guanajuato, Guanajuato, México.

ID $1^{\text {er }}$ Autor: Karina, Galván-Zavala / ORC ID: 0000-0001-5759-8814, arXiv Author ID: 3066602, CVU CONACYT ID: 273545

ID $1^{\text {er }}$ Coautor: Artemio, Jiménez-Rico / ORC ID: 0000-0001-9069-6483, Researcher ID Thomson: S-7880-2018, CVU CONACYT ID: 947479

ID $2^{\text {do }}$ Coautor: Luis Gerardo, Rea-Chavez / ORC ID: 0000-0002-2270-2957, Researcher ID Thomson: 5-8565-2018, CVU CONACYT ID: 947607

\begin{abstract}
Resumen
Resulta importante conocer los avances de armonizacion contable, el consejo nacional de armonización contable presenta para el año 2019 un avance de $76.99 \%$ de cumplimiento en promedio nacional, mientras que el estado de Guanajuato reporta un cumplimiento de $82.49 \%$ mostrando mayor avance de cumplimiento por los sujetos autónomos, esta investigación analiza el cumplimiento al articulo 79 de la ley general de contabilidad gubernamental relativo a la publicación en medios electrónicos del plan de evaluaciones, metodología e indicadores, resultados de las evaluaciones y datos del evaluador. El análisis se aplica para el año 2019 en los organismos autónomos del estado de Guanajuato, resultando solo el $34.12 \%$ de cumplimiento según la métrica de valoración propuesta, lo que evidencia la necesaria sanción por incumplimiento.
\end{abstract}

Evaluacion, presupuesto basado en resultados, Armonizacion contable

\begin{abstract}
It is important to know the advances in accounting, the national accounting council presents for 2019 an advance of $76.99 \%$ compliance on national average, while the state of Guanajuato reports compliance of $82.49 \%$, this research analyses compliance with Article 79 of the General Government Accounting, the publication in electronic media of the evaluation plan, methodology and indicators, evaluation results and evaluator data. The analysis applies for the year 2019 in the autonomous agencies of the state of Guanajuato, resulting in only $34.12 \%$ compliance according to the proposed valuation metric, which shows the necessary penalty for non-compliance.
\end{abstract}

Evaluation, Results-based budget, Accounting

Citación: GALVÁN-ZAVALA Karina, JIMÉNEZ-RICO, Artemio y REA-CHAVEZ, Luis Gerardo. Diagnóstico de programa de evaluación, metodologías, indicadores y evaluación de resultados: caso de organismos autónomos del estado de Guanajuato. Revista de Desarrollo Económico. 2020. 7-22:30-34.

\footnotetext{
$\dagger$ Investigador contribuido como primer autor.
} 


\section{Introducción}

La ley general de contabilidad gubernamental se introdujo en México en el año 2008, tras doce años de implementación e incorporación de la normativa en los procesos contables y presupuestales resulta interesante conocer cuál es el avance, se toma como referencia los datos al año 2019 tras representar un año ya terminado y presentado de información financiera, el Consejo nacional de armonización contable revela que la media de cumplimiento nacional es $82.49 \%$, y Guanajuato se encuentra por debajo de la media nacional con $76.99 \%$, de ello los organismos autónomos son los más avanzados al representar un avance de $99.55 \%$ en cuanto cumplimiento, sin embargo es cuestionable los resultados en cuanto a programa de evaluación al desempeño y resultados de las evaluaciones. Este estudio hace una revisión documental sobre el cumplimiento al artículo 79 de la ley y se presenta un diagnóstico.

\section{Justificación}

El presente documento de investigación pretende identificar el porcentaje de cumplimiento de los organismos autónomos del estado de Guanajuato en referencia al artículo 79 de la ley general de contabilidad gubernamental, relativas a la publicación del programa anual de evaluaciones, la metodología e indicadores de desempeño, los resultados de las evaluaciones realizadas y los datos del evaluador, a efecto de conocer tanto el porcentaje como los sujetos que inciden en un mayor cumplimiento de la norma, en virtud que se tiene identificado por los resultados del Consejo Nacional de Armonización Contable que para el año 2019 los sujetos autónomos del estado de Guanajuato presentaron un cumplimiento del $99.55 \%$ de la Ley en relación con la transparencia y cuenta pública presentada, sin embargo en temas de evaluación de resultados aun presentan un rezago de cumplimiento tanto de programa anual de evaluaciones y resultados de las evaluaciones.

\section{Metodología}

Se examina el año 2019 como año sujeto de revisión, y específicamente “el programa anual de evaluaciones, la metodología e indicadores de desempeño, los resultados de las evaluaciones y los datos del evaluador" normados en el artículo 79 de la Ley General de Contabilidad Gubernamental.
La métrica utilizada es la asignación de $25 \%$ a cada rubro, integrando una ponderación de $100 \%$ a cada uno de los siguientes rubros, se asigna un porcentaje equitativo ya que cada uno de los variables contribuye de igual manera al logro de la evaluación de la armonización contable:

\begin{tabular}{|r|l|r|}
\hline \multicolumn{1}{|c|}{ Rombre anual de } & Ponderación \\
\hline 1 & $\begin{array}{l}\text { Programa } \\
\text { evaluaciones }\end{array}$ & $25 \%$ \\
\hline 2 & $\begin{array}{l}\text { Metodología e indicadores de } \\
\text { desempeño }\end{array}$ & $25 \%$ \\
\hline 3 & $\begin{array}{l}\text { Resultados de las } \\
\text { evaluaciones }\end{array}$ & $25 \%$ \\
\hline 4 & Datos del evaluador & $100 \%$ \\
\hline & Total & \\
\hline
\end{tabular}

Tabla 1

\section{Marco teórico}

Según el artículo 4 fracción I de la ley General de contabilidad Gubernamental se entiende por armonización contable lo siguiente:

\begin{abstract}
"La revisión, restructuración, y compatibilización de modelos contables vigentes a nivel nacional, a partir de la adecuación y fortalecimiento de las disposiciones jurídicas que las rigen, de los procedimientos para el registro de las operaciones, de la información que se deben generar los sistemas de contabilidad gubernamental, y de las características y contenidos de los principales informes de rendición de cuentas;
\end{abstract}

Uno de los objetivos de la armonización contable es la homologación de criterios para el registro de las operaciones que afectan económicamente a la entidad, así como la generación de estados financieros que sean comparables y de los cuales uno de los propósitos fundamentales es contar con una contabilidad nacional y contabilidad comparable entre las diferentes unidades económicas públicas del país. 


\section{Evidencia}

Al año 2020, se cumplen 12 años de trabajos en la implementación de esta Ley General de Contabilidad Gubernamental que tiene entre otros de sus propósitos la transparencia y rendición de cuentas, sin embargo, los desafíos son aún grandes ya que con la última evaluación de cumplimiento se tiene que el promedio nacional de cumplimiento es de $76.99 \%$ caracterizado por un cumplimiento medio.

Calificación general promedio de armonización contable

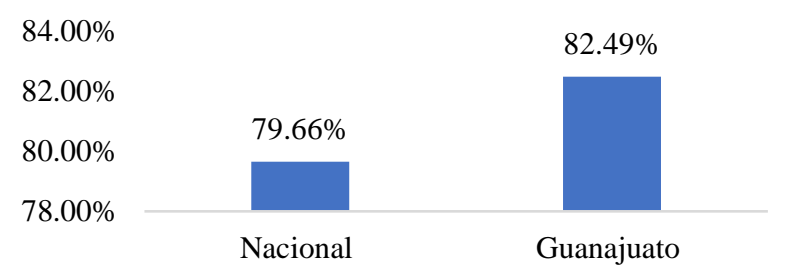

Gráfico 1 Promedio nacional de cumplimiento en la armonización contable

Fuente: Consejo Nacional de Armonización Contable CONAC (2020) disponible en resultados de la evaluación de la armonización contable

Examinando los resultados del estado de Guanajuato en cuanto a transparencia y deuda pública se tiene un cumplimiento de $82.49 \%$, a señalar es superior a la media nacional, pero por debajo de estados como Baja california, Coahuila, Sinaloa, Nayarit, Aguascalientes, Hidalgo, Ciudad de México, tabasco, campeche y Chiapas.

Por otro lado, observando el desglose por tipo de ente público de cumplimiento a la ley general de contabilidad gubernamental se tiene que los poderes legislativo, judicial y órganos autónomos son los sujetos con mayor cumplimiento con un $99.55 \%$ de calificación.

Clasificación genral promedio por tipo...

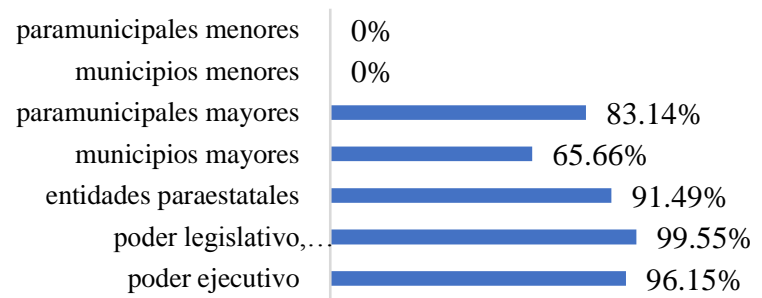

Gráfico 2 Calificación general promedio por tipo de ente público de Guanajuato

Fuente: Consejo Nacional de Armonización Contable (2020) disponible en resultados de la evaluación de la armonización contable
El estado de Guanajuato cuenta con siete organismos autónomos, desglosados de la siguiente manera:

\begin{tabular}{|r|l|}
\multicolumn{1}{|c|}{ Súmero } & \multicolumn{2}{c|}{ Sujeto } \\
\hline 1 & Instituto de Acceso a la Información Pública \\
\hline 2 & $\begin{array}{l}\text { Instituto Electoral del Estado de } \\
\text { Guanajuato. }\end{array}$ \\
\hline 3 & Procuraduría de los Derechos Humanos. \\
\hline 4 & Universidad de Guanajuato \\
\hline 5 & $\begin{array}{l}\text { Tribunal de Justicia Administrativa del } \\
\text { Estado de Guanajuato }\end{array}$ \\
\hline 6 & Tribunal Estatal Electoral de Guanajuato \\
\hline 7 & Fiscalía General del Estado de Guanajuato \\
\hline
\end{tabular}

Tabla 2

Se toma como muestra los organismos autónomos, puesto que por la misma autonomía que les confiere el Estado, la constitución y las leyes que de las cuales emanan, son organismos capaces de implementar de manera ágil las regulaciones, pues cuentan con el presupuesto, la infraestructura, el personal necesario para adoptar y cumplir las regulaciones.

Para delimitar el alcance de este trabajo de investigación nos concentramos en el siguiente instrumento normativo:

Artículo 79.- Los entes públicos deberán publicar en sus páginas de Internet a más tardar el último día hábil de abril su programa anual de evaluaciones, así como las metodologías e indicadores de desempeño. Los entes públicos deberán publicar a más tardar a los 30 días posteriores a la conclusión de las evaluaciones, los resultados de las mismas $e$ informar sobre las personas que realizaron dichas evaluaciones. 
La Secretaría de Hacienda y el Consejo Nacional de Evaluación de la Política de Desarrollo Social, en el ámbito de su competencia y de conformidad con el artículo 110 de la Ley Federal de Presupuesto y Responsabilidad Hacendaria, enviarán al Consejo los criterios de evaluación de los recursos federales ministrados a las entidades federativas, los municipios y los órganos político-administrativos de las demarcaciones territoriales de la Ciudad de México, así como los lineamientos de evaluación que permitan homologar y estandarizar tanto las evaluaciones como los indicadores para que dicho Consejo, en el ámbito de sus atribuciones, proceda a determinar los formatos para la difusión de los resultados de las evaluaciones, conforme a lo establecido en el artículo 56 de esta Ley.

Por otro lado, y de conformidad con el artículo 56 de la LGCG, tenemos lo siguiente:

Artículo 56.- La generación y publicación de la información financiera de los entes públicos a que se refiere este Título, se hará conforme a las normas, estructura, formatos y contenido de la información, que para tal efecto establezca el consejo y difundirse en la página de Internet del respectivo ente público. Dicha información podrá complementar la que otros ordenamientos jurídicos aplicables ya disponen en este ámbito para presentarse en informes periódicos y en las cuentas públicas. Asimismo, la información se difundirá en los medios oficiales de difusión en términos de las disposiciones aplicables.

\section{Resultados}

En el año 2012, para ser exactos el pasado 12 de noviembre de 2012 se adicionó a la ley el capítulo $\mathrm{V}$ de la información financiera relativa a la evaluación y rendición de cuentas, mientras que el artículo 79 se reformó el 30 de diciembre de 2015 y posteriormente el 19 de enero de 2018. Esto con la intención de que el sujeto público difunda su programa anual de evaluaciones, la metodología e indicadores de desempeño, así como el resultado de las evaluaciones, indicando también los sujetos evaluadores.
De tal manera que, para este estudio se toma muestra del $100 \%$ de los organismos autónomos de la entidad federativa de Guanajuato para evaluar el nivel de cumplimiento del referido artículo 79 de la LGCG, teniendo como resultado solo el $32.14 \%$ de acuerdo con la siguiente tabla de cumplimiento.

\begin{tabular}{|c|c|c|}
\hline Institución & $\begin{array}{c}\text { Cumplimiento } \\
\text { del artículo } 79 \\
\text { LGCG para el } \\
\text { año } 2019\end{array}$ & $\begin{array}{c}\text { Semaforización } \\
\text { de acuerdo con } \\
\text { la información } \\
\text { publicada }\end{array}$ \\
\hline $\begin{array}{lr}\text { Instituto de } \\
\text { Acceso a la } \\
\text { Información } \\
\text { Pública. }\end{array}$ & $\begin{array}{l}\text { Metodologías e } \\
\text { indicadores de } \\
\text { desempeño }\end{array}$ & $25 \%$ \\
\hline $\begin{array}{l}\text { Instituto } \\
\text { Electoral del } \\
\text { Estado de } \\
\text { Guanajuato. } \\
\end{array}$ & $\begin{array}{l}\text { Metodologías e } \\
\text { indicadores de } \\
\text { desempeño }\end{array}$ & $25 \%$ \\
\hline $\begin{array}{l}\text { Procuraduría de } \\
\text { los Derechos } \\
\text { Humanos }\end{array}$ & $\begin{array}{l}\text { Metodologías e } \\
\text { indicadores de } \\
\text { desempeño }\end{array}$ & $25 \%$ \\
\hline $\begin{array}{l}\text { Universidad de } \\
\text { Guanajuato }\end{array}$ & $\begin{array}{l}\text { Metodologías e } \\
\text { indicadores de } \\
\text { desempeño }\end{array}$ & $25 \%$ \\
\hline $\begin{array}{l}\text { Tribunal de } \\
\text { Justicia } \\
\text { Administrativa } \\
\text { del Estado de } \\
\text { Guanajuato }\end{array}$ & $\begin{array}{l}\text { Metodologías e } \\
\text { indicadores de } \\
\text { desempeño }\end{array}$ & $25 \%$ \\
\hline $\begin{array}{l}\text { Tribunal Estatal } \\
\text { Electoral de } \\
\text { Guanajuato }\end{array}$ & $\begin{array}{l}\text { Programa anual } \\
\text { de evaluaciones } \\
\text { Metodologías e } \\
\text { indicadores de } \\
\text { desempeño } \\
\text { Resultados de } \\
\text { las evaluaciones } \\
\text { Datos del } \\
\text { evaluador }\end{array}$ & $100 \%$ \\
\hline $\begin{array}{lr}\text { Fiscalía } & \\
\text { General del } \\
\text { Estado } \\
\text { Guanajuato } \\
\end{array}$ & $\begin{array}{l}\text { No presenta } \\
\text { información } \\
\text { pública }\end{array}$ & $0 \%$ \\
\hline
\end{tabular}

Tabla 3 "Cumplimiento al artículo 79 LGCG por parte de los sujetos autónomos del estado de Guanajuato" Fuente: elaboración propia a partir de información púbica contenida en los portales electrónicos de los sujetos en revisión

De la tabla anterior se desprende que el promedio de cumplimiento es $32.14 \%$ de cumplimiento en relación con el artículo 79 de la Ley general de contabilidad gubernamental. 


\section{Conclusiones}

El consejo nacional de armonización contable presenta para el año 2019 un avance de $76.99 \%$ de cumplimiento en promedio nacional, mientras que el estado de Guanajuato reporta un cumplimiento de $82.49 \%$ mostrando mayor avance de cumplimiento por los sujetos autónomos, analizando el artículo 79 de la ley general de contabilidad gubernamental relativo a la publicación en medios electrónicos del plan de evaluaciones, metodología e indicadores, resultados de las evaluaciones y datos del evaluador, se detectó solo el $34.12 \%$ de cumplimiento de los organismos autónomos del estado de Guanajuato según la métrica de valoración propuesta, lo que evidencia la necesaria sanción por incumplimiento.

\section{Referencias}

Consejo Nacional de Armonización Contable (2020) "normatividad del consejo nacional de armonización contable" recuperado el 29 de febrero de 2020 de https://www.conac.gob.mx/es/CONAC/Normati vidad_Vigente

José Armando Plata Sandoval. (2016). Implementación de la armonización contable gubernamental en México. El Cotidiano, 32(198), 27.

Ley de disciplina financiera de las entidades federativas y los municipios: http://www.diputados.gob.mx/LeyesBiblio/pdf/ LDFEFM_300118.pdf

Ley general de contabilidad gubernamental: http://www.diputados.gob.mx/LeyesBiblio/pdf/ LGCG_300118.pdf

MIRANDA, P. E. (2011). contabilidad gubernamental. caso práctico contable $y$ presupuestal Ediciones Fiscales ISEF, SA.

Rico, Cornelio. (2019). "cierre contable y presupuestal de la contabilidad gubernamental", instituto mexicano de contadores públicos. México

Rico, Cornelio. (2019). "contabilidad gubernamental y su información financiera", instituto mexicano de contadores públicos. México. 